\title{
Foreign Body in Gastrointestinal Tract
}

National Cancer Institute

\section{Source}

National Cancer Institute. Foreign Body in Gastrointestinal Tract. NCI Thesaurus. Code

C34622.

Extraneous material located within the gastrointestinal tract. 